\title{
Chemical ordering in PtNi nanocrystals
}

\author{
Okkyun Seo ${ }^{\mathrm{a}}$, Ji Yeon Lee ${ }^{\mathrm{a}}$, Jae Myung Kim ${ }^{\mathrm{a}}$, Jin-Woo Kim ${ }^{\mathrm{a}}$, Hyon Chol \\ Kang ${ }^{\mathrm{b}}$, Jinwook Chung ${ }^{\mathrm{c}}$, Do Young Noh ${ }^{\mathrm{a}, *}$ \\ ${ }^{a}$ Department of Physics and Photon Science \& School of Materials Science and \\ Engineering, Gwangju Institute of Science and Technology, Gwangju 61005, Korea \\ ${ }^{b}$ Department of Advanced Materials Engineering, Chosun Univ., Gwangju 61542, Korea \\ ${ }^{c}$ Department of Physics, Pohang Univ. of Science and Technology, Pohang 37673, Korea
}

\begin{abstract}
We investigated the chemical ordering in PtNi nanocrystals fabricated on sapphire substrate using in-situ synchrotron x-ray scattering. Nanocrystals with composition close to $1: 1$ were ordered in the tetragonal $\mathrm{L} 1_{0}$ structure at low temperatures. The transition to disordered FCC structure occurred at around $640{ }^{\circ} \mathrm{C}$ and substantial hysteresis of about $50 \mathrm{~K}$ was observed. Nanocrystals of smaller sizes fabricated under the same conditions were Ni rich and ordered into $\mathrm{Cu}_{3} \mathrm{Au}$ type $\mathrm{L}_{2}$ structure. Significantly higher degree of chemical ordering was observed in $\mathrm{L} 1_{2}$ structure than in $\mathrm{L}_{0}$ structure.
\end{abstract}

Keywords: PtNi alloy nanocrystals, chemical ordering, order-disorder transition, $\mathrm{L} 1_{0} \& \mathrm{~L} 1_{2}$ structure

\section{Introduction}

Recently, Pt based bimetallic alloy nanoparticles attracted much attention for applications in fuel cell electrodes and high density magnetic storage devices 1, [2, 3, 4, since the catalytic activity can be optimized and the amount of Pt can be 5 reduced by alloying with less expensive metals. Among the Pt bimetallic alloys for fuel cell applications, PtNi nanocrystals have been extensively investigated due to their excellent oxygen reduction reaction efficiency (ORR) surpassing Pt. For example, the ORR of $\mathrm{Pt}_{3} \mathrm{Ni}$ nanoparticles was reported to be much higher than Pt [5, 6]. Pt-Ni core-shell structures have been synthesized and shown to 10 enhanced the catalytic activity for ORR [7. PtNi nanoparticles exhibit only weak ferromagnetic or paramagnetic behavior different from other Pt bimetallic alloys such as PtCo and PtFe, and they are mostly considered for the catalytic applications rather than magnetic applications. Tunability in size, shape, alloy composition, and elemental distribution of $\mathrm{Pt}$ alloy nanocrystals provides a great opportunity to optimize desired specific properties for specific applications.

\footnotetext{
* Corresponding author

Email address: dynoh@gist.ac.kr (Do Young Noh)
}

Preprint submitted to Journal of Alloys and Compounds

December 31, 2015

(C) 2016. This manuscript version is made available under the Elsevier user license http://www.elsevier.com/open-access/userlicense/1.0/ 
Bulk Pt based bimetallic alloys are known to exhibit chemical ordering below order-disorder transition temperature $\left(T_{C}\right)$ in tetragonal $\mathrm{L} 1_{0}$ or cubic $\mathrm{L} 1_{2}$ structure depending on the alloy composition [8, 9. Tetragonal $\mathrm{L} 1_{0}$ structure is preferred when the compositions of $\mathrm{Pt}$ and alloying metals are similar. Above

${ }_{20} T_{C}$, the alloys are chemically disordered and alloying elements are randomly distributed on FCC lattice sites. Structural behaviors including melting and ordering of $\mathrm{Pt}$ bimetallic alloys in a nanocrystal form might be different from bulk due to the effects of finite size. It has been reported that Pt alloy nanocrystals in a chemically ordered structure exhibit distinct magnetic properties and superior

25 catalytic activities $10,11,12,13$. Theoretical studies predicted that the nature of chemical ordering changes as the particle size decreases to a few nm scale 14, 15. Understanding the role of surface atoms and the interplay between the morphology and atomic ordering is important in nanocrystals. [16. Recent Monte Carlo and molecular dynamics study showed that the $\mathrm{L} 1_{0}$ structure in

30 $\mathrm{PtNi}$ nanoparticles is very stable and short range order persists to very high temperatures 17 .

Experimental studies on structure and chemical ordering in alloy nanocrystals such as order-disorder transition and phase segregation have been difficult due to their small sizes and complicated behaviors. Nanocrystals in powder form

35 tends to agglomerate at high temperatures and easily oxidized under non vacuum environment. Distinguishing equilibrium thermodynamic behavior from kinetically limited behavior becomes difficult as the size of particles approach nanometer scale. Due to these reasons, experimental data on detailed structural properties including atomic ordering in alloy nano crystals are still lacking.

40 In this report, we investigated the atomic ordering of PtNi nanocrystals aligned on a sapphire substrate using the in-situ synchrotron x-ray scattering technique. Nanocrystals with size above $150 \mathrm{~nm}$ exhibited a transition from the ordered $\mathrm{L} 1_{0}$ structure to disordered FCC structure around $650{ }^{\circ} \mathrm{C}$. We observed a temperature hysteresis of about $50 \mathrm{~K}$ in the order parameter and lattice struc-

45 ture across the transition, which was not reported previously. Nanocrystals of smaller sizes were $\mathrm{Ni}$ rich and exhibited much higher degree of ordering. The crystal structure of the ordered phase was cubic $\mathrm{L} 1_{2}$ rather than the tetragonal $\mathrm{L} 1_{0}$.

\section{Experimental Details}

50

The PtNi alloy nanocrystals were prepared by applying the solid-state dewetting process on Pt-Ni bilayer films which were grown on a sapphire (0001) substrate by electron beam evaporation at room temperature. The thickness ratio of each layer in the Pt-Ni bilayer were maintained to 1:1, while each layer thickness was varied to obtain $\mathrm{PtNi}$ alloy nano crystals of various sizes. In this 55 experiment, we report the results from $\mathrm{Pt}-\mathrm{Ni}$ bilayer films with layer thickness of $1,2.5$, and $5 \mathrm{~nm}$. PtNi alloy nanocrystals were obtained by rapid thermal annealing (RTA) of the Pt-Ni bilayer films to $1050^{\circ} \mathrm{C}$ for 10 minutes. The ramping rate of $\mathrm{RTA}$ was about $200{ }^{\circ} \mathrm{C} / \mathrm{min}$. The bilayer films were transformed into 

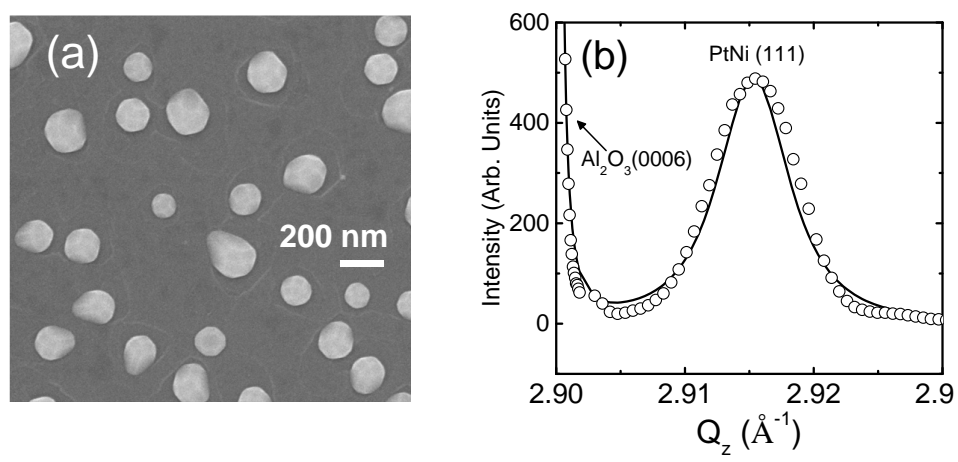

Figure 1: (a) SEM image of PtNi nanocrystals obtained by annealing a $\mathrm{Pt}_{5 \mathrm{~nm}}-\mathrm{Ni}_{5 \mathrm{~nm}}$ bilayer film. (b) X-ray diffraction profile of the PtNi (111) Bragg peak. The sharp rise in the left side is the tail of the substrate sapphire (0006) Bragg peak. The line is the result of a fit to estimate the peak width.

completely mixed disordered alloy nanocrystals through solid-state dewetting process during annealing 18 .

To transform the nanocrystals into chemically ordered structure, we annealed the samples at $530{ }^{\circ} \mathrm{C}$ below the expected order-disorder transition temperature for 50 hours. The $\mathrm{Pt}$ ratio in the nanocrystals, estimated from the unit cell size by applying the Vegard's law, varied from about 0.54 to 0.37 depending 65 on the crystal size. The crystal orientation of the PtNi nanocrystals thus obtained were well aligned to the substrate sapphire orientation, which facilitated the structural analysis using $\mathrm{x}$-ray diffraction significantly. The $<111>$ axis of the PtNi nanocrystals were well aligned to the sapphire $<0001>$ direction, while the in-plane $<11 \overline{2}>$ were parallel to the sapphire $<11 \overline{2} 0>$ direction.

The lateral particle size and size distribution were investigated by scanning electron microscopy (SEM) as illustrated in Fig. 1(a) which shows the PtNi nanocrystals obtained by annealing a $\mathrm{Pt}_{5 \mathrm{~nm}}-\mathrm{Ni}_{5 \mathrm{~nm}}$ film. The diameter of the nanocrystals was $160 \pm 50 \mathrm{~nm}$. The particle size in the vertical direction, estimated from the width of the (111) Bragg peak shown in Fig. 1(b) using Scher-

75 rer's equation, was about $80 \mathrm{~nm}$, which is about half the lateral size. As the size of nanocrystals decreases below $200 \mathrm{~nm}$, the crystal domain size estimated by the width of the Bragg peaks approaches the physical height of nanocrystals. Typically the vertical height of the nanocrystals supported on sapphire substrates is about half the lateral size 18 .

so We carried out the in-situ synchrotron x-ray scattering experiments at $5 \mathrm{D}$ GIST beam-line of Pohang Light Source (PLS) in Korea and at beamline X20 at National Synchrotron Light Source(NSLS) in USA. The x-ray energy was fixed at $10 \mathrm{keV}$ by using a double-bounce Si (111) monochromator and focused by an x-ray mirror. The samples were maintained in a temperature controlled

85 chamber with an $\mathrm{x}$-ray window maintained at the $3 \times 10^{-5}$ Torr of vacuum. The chemical ordering was investigated using the superlattice (100) peak which is forbidden in the disordered FCC structure. To investigate the crystal structure, 


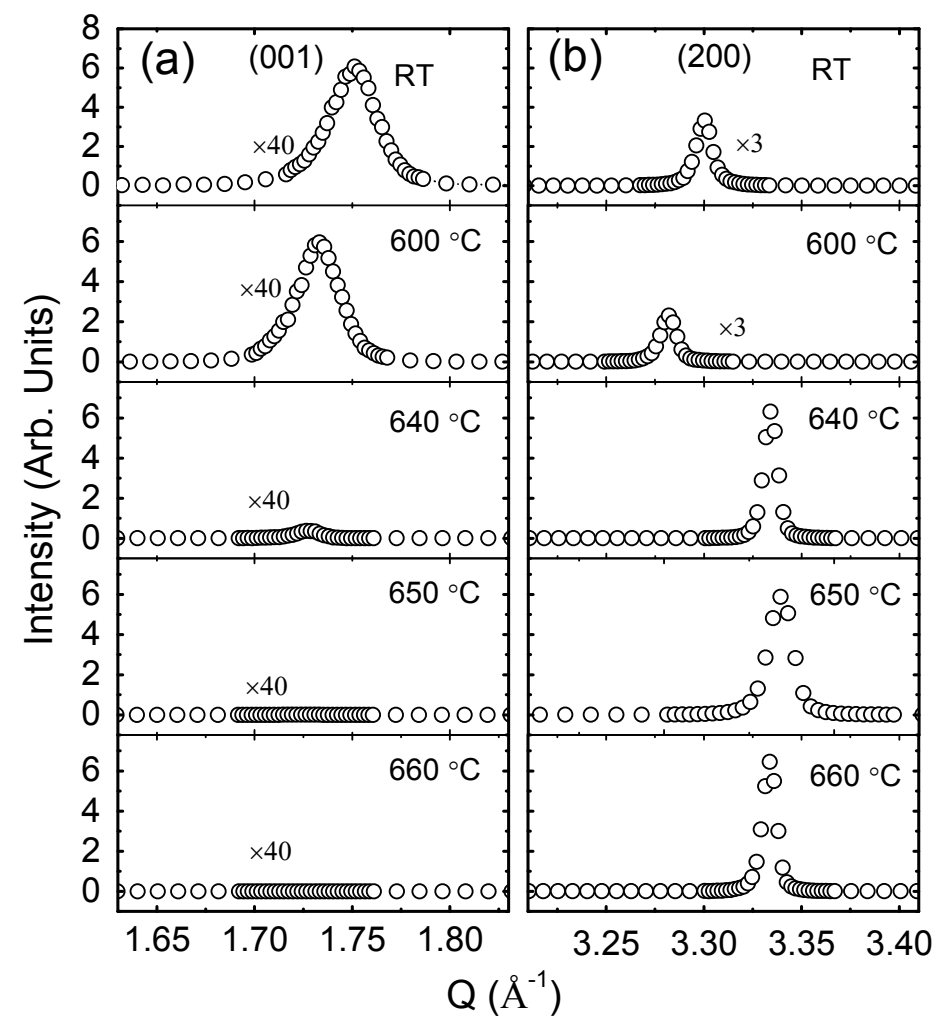

Figure 2: In-situ x-ray diffraction profile of the superlattice (001) reflection (a) and the fundamental (200) Bragg reflection (b) obtained from the PtNi nanocrystals at various temperatures across the order-disorder transition.

we measured the fundamental (200) peak as well as the (111) Bragg peak in the substrate normal direction.

$90 \quad$ The order-disorder transition was investigated by measuring the (100) and the (200) peak as we vary the sample temperature across the transition temperature $T_{C}$. X-ray diffraction profiles were measured at each temperature after waiting for $30 \mathrm{~min}$ to establish equilibrium. Starting from the ordered structure at room temperature, the measurement was done first as the sample was heated to across $T_{C}$ (heating run). The measurement was repeated during cooling the sample below $T_{C}$ (cooling run). The reproducibility was confirmed by performing a second heating run following the cooling run.

\section{Results and Discussion}

\subsection{Order-Disorder transition in PtNi nanocrystals in $L 1_{0}$ structure}

100

We first present the order-disorder transition investigated on the PtNi nanocrystals obtained by annealing a $\mathrm{Pt}_{5 n m}-\mathrm{Ni}_{5 n m}$ film (Sample \#1) whose average lat- 
eral (vertical) size was about $160(80) \mathrm{nm}$. The Pt composition estimated from the unit cell volume was about 0.54. As signified by the well-defined sharp superlattice (001) peak in the longitudinal direction measured at room temperature illustrated in Fig. 2(a), the nanocrystals showed high degree of chemical ordering. The size of the chemically ordered domain or the correlation length of the chemical ordering estimated from the half width at the half maximum (HWHM) of the peak, was about $21 \mathrm{~nm}$. We also measured the fundamental (200) Bragg peak, shown in Fig. 2(b), which occurred at a momentum transfer 110 value smaller than the twice the (001) peak position. This indicates that the unit cell was elongated along the [100] direction and the ordered structure was the tetragonal $\mathrm{L} 1_{0}$. Together with the position of the (111) Bragg peak shown in Fig. 1(b), we obtained the unit cell dimensions in all direction. The fundamental peak was sharper than the superlattice peak and the crystal domain size 15 estimated from the HWHM of the fundamental peak was about $60 \mathrm{~nm}$. We conclude that one crystal domain included two to four ordered domains separated by domain boundaries.

The order-disorder transition occurred at around $640{ }^{\circ} \mathrm{C}$ as illustrated in the data shown in Fig. 2. As the sample temperature was increased to $600{ }^{\circ} \mathrm{C}$, 120 the (001) peak becomes a little sharper and shifted towards the low-Q direction indicating that the lattice was expanded and chemical ordering was improved. This improvement was due to the annealing effect, although the change was minimal. At $640{ }^{\circ} \mathrm{C}$, however, the (001) superlattice peak intensity suddenly decreased and the fundamental (200) peak shifted to a higher-Q value with 125 increased intensity. This indicates that the chemical ordering was lost and the crystal structure was changed. Above $T_{C}$, the crystal structure was found to be cubic FCC as confirmed by the lattice constants evaluated using the peak positions of the (200) and the (111) Bragg peak.

The order-disorder transition in the PtNi nanocrystals was a first order tran-

130 sition with a large temperature hysteresis. Figure 3 illustrates the behavior of the order parameter evaluated from the superlattice (001) peak intensity and the $a$ - and $c$-axis lattice parameter evaluated from the fundamental (200) and (111) peak positions.

The order parameter was defined as the the sum of the fraction of Pt sites occupied by Pt atoms and the fraction of Ni sites occupied by Ni atoms [19]. It is zero for a completely random system and unity for completely ordered system. The order parameter S presented in Fig. 3 was evaluated from the ratio of the integrated intensity of the superlattice (001) peak, $I_{s}$ and the fundamental (200) peak, $I_{f}$ assuming that the $\mathrm{CuAu}$ type of chemical ordering. Specifically the following formula,

$$
S^{2}=\frac{I_{s}}{I_{f}}\left(\frac{L_{f} D_{f}}{L_{s} D_{f}}\right)\left(\frac{F_{P t}+F_{N i}}{F_{P t}-F_{N i}}\right)^{2},
$$

where $L_{f}(s)$ and $D_{f}(s)$ are Lorentz factor and Debye-Waller factor for the fun135 damental (superlattice) peaks, and $F_{P t}$ and $F_{N i}$ are atomic form factor of $\mathrm{Pt}$ and $\mathrm{Ni}$ respectively. 


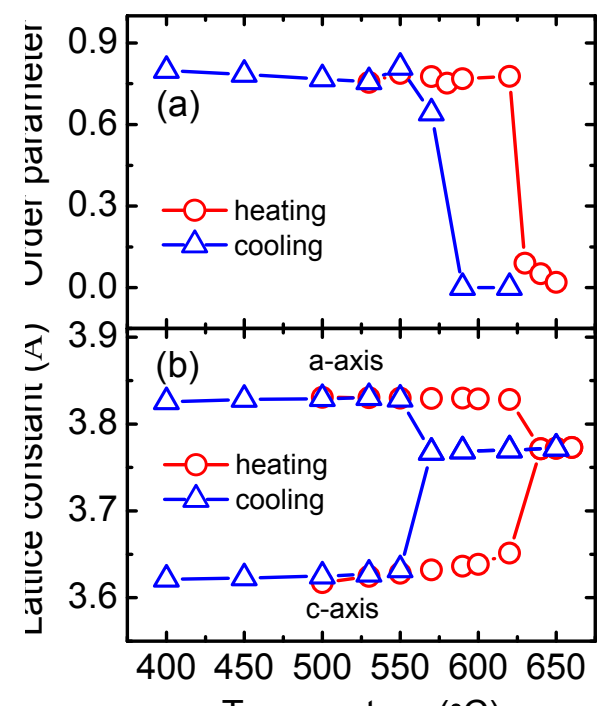

Figure 3: (a) Order parameter representing the chemical order in the PtNi nanocrystals evaluated from the superlattice (100) peak. (b) $a$ - and $c$-axis constants evaluated from the fundamental (200) and (111) Bragg reflection. Broad hysteresis was observed between the heating and cooling run. The lines are guide-to-the-eyes.

Abrupt changes were observed both in the order parameter and in the lattice structure during heating and cooling run indicating that the transition was firstorder. We observed a large hysteresis of about $50 \mathrm{~K}$, that is, the transition during the cooling run occurred about $50 \mathrm{~K}$ below the transition temperature observed during the heating run. Although we cannot completely exclude the effect of kinetic limitations, the hysteresis observed in this work was rather large.

\section{2. $L 1_{2}$ ordered structure observed in $\mathrm{Ni}$ rich PtNi nanocrystals}

PtNi nanocrystals obtained from thin $\mathrm{Pt}_{2.5 \mathrm{~nm}}-\mathrm{Ni}_{2.5 \mathrm{~nm}}$ (Sample \#2) and $\mathrm{Pt}_{1 n m}-\mathrm{Ni}_{1 n m}$ (Sample \#3) bilayer films exhibited chemical ordering different from Sample \#1. The average lateral size of the nanoparticles in Sample \#2 and \#3 estimated from SEM images was about $120 \mathrm{~nm}$. Shown in Fig. 4 are the superlattice (001) peak obtained from Sample \#2 and \#3 at room temperatures together with that from Sample \#1 discussed in the previous section. First, we note that the (001) peaks of Sample \#2 and \#3 shifted towards the lowQ side significantly indicating that their unit cell was larger than Sample \#1 in this direction. The fundamental (200) and (111) peak positions were also different from those of Sample \#1 (data not shown). By evaluating the unit cell dimensions, we found that the crystal structure was close to cubic within $1 \%$ as

155 summarized in table 1 . From the unit cell dimensions, we found that chemical ordered nanocrystals in Sample \#2 and \#3 were Ni-rich with reduced Pt ratio of about 0.38. Presumably, $\mathrm{Pt}$ atoms are segregated out to achieve a stable 


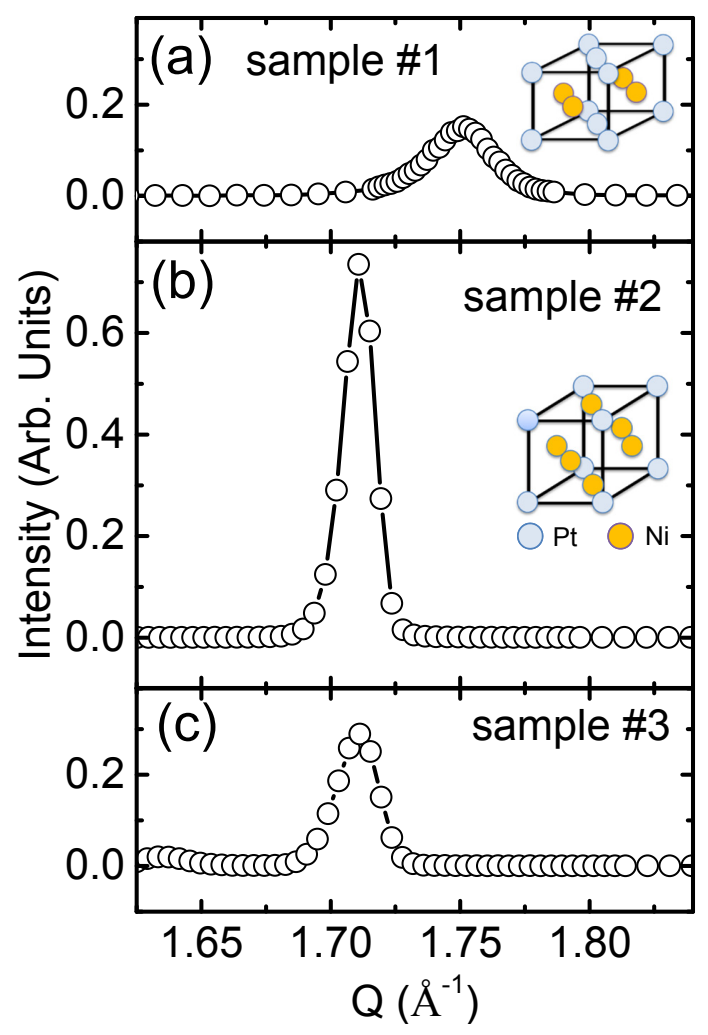

Figure 4: Superlattice (001) Bragg peak of Sample \#1 (a), \#2(b), and \#3 (c) measured at room temperature. The ordered $\mathrm{L}_{0}$ (a) and $\mathrm{L}_{2}$ (b) structures are illustrated as insets. The lines are guide-to-the-eyes.

ordered configuration during long annealing to obtain the ordered structure. From the data shown in table 1, we conclude that Sample \#2 and \#3 were ordered in $\mathrm{Cu}_{3} \mathrm{Au}$ type cubic $\mathrm{L} 1_{2}$ structure rather than the tetragonal $\mathrm{L} 1_{0}$.

The superlattice (001) peak of Sample \#2 and \#3 was also much sharper and more intense indicating that the degree of chemical ordering was higher and the ordered domains were larger. The ordered domain size estimated from the HWHM of the superlattice peak was 40 and $31 \mathrm{~nm}$ respectively, which is larger than the domain size of Sample \#3, $21 \mathrm{~nm}$, in $\mathrm{L} 1_{0}$ structure. We think that the order domain size in these samples was close to the particle dimension in the $<001>$ direction, and most nanocrystals were consisted with single ordered domain. The high degree of ordering and large domains were possible in the cubic $\mathrm{L}_{2}$ structure because there is no distinction between the $a$-type and $c$ type domains any more. 
Table 1: Lattice constants, crystal structure, and composition

\begin{tabular}{cccll}
\hline sample & $\mathrm{a}=\mathrm{b}(\AA)$ & $\mathrm{c}(\AA)$ & structure & composition \\
\hline$\# 1$ & 3.808 & 3.595 & $\mathrm{~L} 1_{0}$ (tetragonal) & $P t_{0.54} N i_{0.46}$ \\
$\# 2$ & 3.671 & 3.684 & $\mathrm{~L}_{2}$ (cubic) & $P t_{0.39} N i_{0.61}$ \\
$\# 3$ & 3.672 & 3.663 & $\mathrm{~L}_{2}$ (cubic) & $P t_{0.37} N i_{0.63}$ \\
\hline
\end{tabular}

\section{Conclusions}

In this work, we investigated the chemical ordering in PtNi nanocrystals aligned on a sapphire substrate using in-situ x-ray scattering. Relatively large nanocrystals with composition close to $1: 1$ were ordered in tetragonal $\mathrm{L} 1_{0}$ crys-

175 tal structure. The order-disorder transition was first order. A large thermal hysteresis of about $50 \mathrm{~K}$ was observed both in the order parameter and in the lattice structure, which, we think, is unusually large. Nanocrystals of smaller size became $\mathrm{Ni}$ rich during the ordering process and exhibited cubic $\mathrm{L} 1_{2}$ ordered structure of $\mathrm{Cu}_{3} \mathrm{Au}$ type. The chemical ordering was superb and the ordered domain size was close to the size of nanocrystals.

As the particle size decreases below $100 \mathrm{~nm}$, they tends to phase-separate and $\mathrm{Ni}$ rich particles would appear. When the composition deviates from 1:1 toward 3:1, we expect that PtNi nanocrystals exhibit the Cu3Au type of ordering in L12 structure. Although the transition temperature of particles close to $100 \mathrm{~nm}$ is similar to the bulk value, higher transition temperature predicted in literatures might appear in much smaller particles. Further studies in smaller nanocrystals would be useful for complete understanding of the size and composition dependence of the chemical ordering in Pt bimetallic nano alloy crystals.

\section{Acknowledgments}

We acknowledge the support and fruitful discussions provided by Dr. J.J. Sweet at NSLS and Dr. O. Sakata at Spring-8. This work was supported by the National Research Foundation of Korea (NRF) grant funded by the Korean government (MSIP) through SRC (NRF-2015R1A5A1009962), and We also acknowledge the GSG Project through a grant provided by GIST in 2015 and the support by Institute for Basic Science (IBS).

\section{References}

[1] C. Wang, G. Wang, D. van der Vilet, K.-C. Chang, N. M. Markovic, V. R. Stamenkovic, Phys. Chem. Chem. Phys. 12 (2010) 6933.

[2] T. Toda, H. Igarashi, H. Uchida, M. Watanabe, J. Electrochem. Soc. 146 (1999) 3750.

[3] F. Tournus, A. Tamion, N. Blanc, A. Hannour, L. Bardotti, B. Prevel, P. Ohresser, E. Bonet, T. Epicier, V. Dupuis, Phys. Rev. B 77 (2008) 144411. 
[4] Y. Yu, W. Yang, X. Sun, W. Zhu, X.-Z. Li, D. J. Sellmyer, S. Sun, Nano Lett. 14 (2014) 2778.

[5] H. Yang, W. Vogel, C. Lamy, N. Alonso-Vante, J. Phys. Chem. B 108 (2004) 11024.

[6] V. R. Stamenkovic, B. Fowler, B. S. Mun, G. Wang, P. N. Ross, C. A. Lucas, N. M. Markovic, Science 315 (2007) 493.

[7] G. Wang, H. Wu, D. Wexler, H. Liu, O. Savadogo, J. Alloys and Compd 503 (2010) L1-L4.

[8] C. E. Dahmani, M. C. Cadeville, J. M. Sanchez, J. L. Moran-Lopez, Phys. Rev. Lett. 55 (1985) 1208.

[9] Y. L. Bouar, A. Loiseau, A. Finel, Phys. Rev. B 68 (2003) 224203.

[10] Y. J. Zhang, Y. T. Yang, Y. Liu, Y. X. Wang, L. L. Yang, M. B. Wei, H. G. Fan, H. J. Zhai, X. Y. Liu, Y. Q. Liu, N. N. Yang, Y. H. Wu, J. H. Yang, J. Phys. D:Appl. Phys. 44 (2011) 295003.

[11] M. Chen, D. E. Nikles, J. Appl. Phys. 91 (2002) 8477.

[12] L. Xiong, A. Manthiram, J. Electrochem. Soc. 152 (2005) A697.

[13] S. Koh, M. F. Toney, P. Strasser, Electrochim. Acta 52 (2007) 2765.

[14] D. Alloyeau, C. Ricolleau, C. Mottet, T. Oikawa, C. Langlois, Y. L. Bouar, N. Braidy, A. Loiseau, Nat. Mater. 8 (2009) 940.

[15] W. Qi, Y. Li, S. Xiong, S.-T. Lee, Small 6 (2010) 1996.

[16] D. Cheng, D. C. W. Wang, S. Huang, J. Phys. Chem. C 112 (2008) 4855.

225 [17] D. Cheng, S. Yuan, R. Ferrando, J. Phys: Condens. Matter 25 (2013) 355008 .

[18] O. Seo, S. A. Oh, J. Y. Lee, S. S. Ha, J. M. Kim, J. W. Choi, J.-W. Kim, H. C. Kang, D. Y. Noh, J. Alloys Compd. submitted for publication.

[19] B. E. Warren, X-ray Diffraction, Addison-Wesley Publishing Co, Inc., Reading, Mass., USA, 1990. 